\title{
Locking of El Niño's Peak Time to the End of the Calendar Year in the Delayed Oscillator Picture of ENSO
}

\author{
ELI TZIPERMAN \\ Department of Environmental Sciences, Weizmann Institute of Science, Rehovot, Israel \\ Mark A. Cane, Stephen E. Zebiak, Yan Xue, and B. Blumenthal \\ Lamont-Doherty Earth Observatory, Columbia University, Palisades, New York
}

(Manuscript received 10 November 1996, in final form 7 October 1997)

\begin{abstract}
El Niño events owe their name to their tendency to be locked to the seasonal cycle. A simple explanation is proposed here for the locking of the peak of ENSO's basin-scale warming to the end of the calendar year. The explanation is based on incorporating a seasonally varying coupled ocean-atmosphere instability strength into the delayed oscillator mechanism for the ENSO cycle. It is shown that the seasonally varying amplification of the Rossby and Kelvin ocean waves by the coupled instability forces the events to peak when this amplification is at its minimum strength, at the end of the calendar year. The mechanism is demonstrated using a simple delayed oscillator model and is further analyzed using the Cane-Zebiak model. Being based on the oversimplified delayed oscillator paradigm of ENSO, the proposed mechanism cannot be expected to fully explain the locking of observed events to the end of the year. However, the wave dynamics perspective it offers to approaching the ENSO phase-locking problem may serve as a first step toward a fuller explanation based on more realistic models and additional data analysis.
\end{abstract}

\section{Introduction}

The phase locking of El Niño-Southern Oscillation (ENSO) events to the seasonal cycle (Rasmusson and Carpenter 1982) is an indication that the seasonal cycle plays an important role in ENSO's dynamics. Figure 1 shows superimposed NINO3 time series from most of the observed ENSO events over the past $100 \mathrm{yr}$. The few events that did not peak near the end of the calendar year, notably the recent events during the 1990s, are not plotted. Still, the plotted majority of observed events clearly demonstrate the robustness of the locking of the events peak time, as measured by the peak time of the NINO3 index, to the end of the calendar year. Recent theories (Tziperman et al. 1994; Jin et al. 1994; Chang et al. 1994; Tziperman et al. 1995; Chang et al. 1995; see also Vallis 1988) indeed attribute ENSO's irregularity and phase locking to a chaos mechanism forced by the seasonal cycle in the equatorial Pacific. However, the mechanism by which the events' peak time is forced to occur primarily toward the end of the year is not yet understood.

Corresponding author address: Dr. Eli Tziperman, Department of Environmental Sciences, Weizmann Institute of Science, 76100 Rehovot, Israel.

E-mail: eli@beach.weizmann.ac.il
We propose here a simple mechanism for ENSO's locking to the end of the calendar year, based on incorporating a seasonally varying coupled ocean-atmosphere instability strength (Zebiak and Cane 1987, hereafter CZ) into the delayed oscillator mechanism (Suarez and Schopf 1988; Graham and White 1988; Battisti 1988; Cane et al. 1990; Munnich et al. 1991) for the ENSO cycle. We show that the seasonally varying amplification of the Rossby and Kelvin ocean waves by the coupled instability forces the events to peak when this amplification is at its minimum strength, at the end of the calendar year. The delayed oscillator model used here assumes (unrealistically) that the SST adjustment time is negligible, and that the ocean memory is due to the equatorial waves travel time only. We therefore do not expect this highly simplified delayed oscillator mechanism to fully explain the locking of actual observed events to the end of the year. However, the wave dynamics perspective offered by this mechanism may help in formulating a fuller explanation based on more realistic models and additional data analysis.

It has been known for quite some time that the strength of the coupled instability, which is responsible for the onset of ENSO events, varies seasonally. Several possible factors may induce stronger instability at various times of the year, among them the intertropical convergence zone (ITCZ) being near the equator (Phi- 


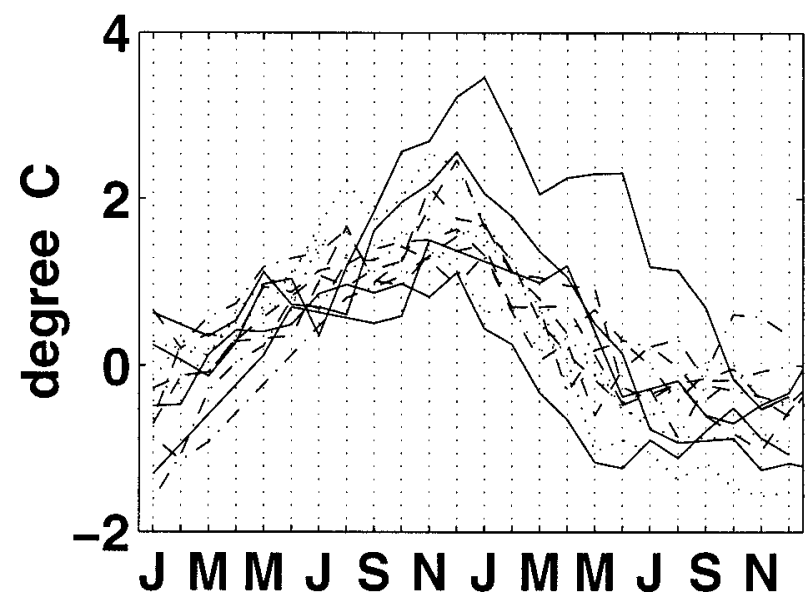

FIG. 1. Two-year segments of the observed NINO3 index (SST averaged over $5^{\circ} \mathrm{S}-5^{\circ} \mathrm{N}$ and $90^{\circ}-150^{\circ} \mathrm{W}$ in the eastern Pacific) during observed ENSO events. SST data is from the Jan 1993 version of the Global Ocean Surface Temperature Atlas (GOSTA, Bottomley et al. 1990). The plotted segments begin at Jan of the years 1888 , 1896, 1902, 1911, 1925, 1930, 1951, 1957, 1963, 1965, 1972, 1976, and 1982 .

lander 1983; Hirst 1986), large mean horizontal SST gradients, shallow thermocline, strong winds, high SST (Hirst 1986), and strong upwelling (Battisti 1988). Each of these factors amplifies the coupled instability during a different time of year. Together, they cause the instability to be strongest during boreal spring and summer (Philander 1983; Hirst 1986), and weakest during early winter. The seasonal variation of the background stability in the equatorial Pacific was noted and investigated in many studies of ENSO predictability (Blumenthal 1991; Goswami and Shukla 1991; Xue et al. 1994; Latif et al. 1994; Chen et al. 1995; Battisti and Sarachik 1995).

The strong instability during spring and early summer was suggested as an explanation for the observation that ENSO's onset typically occurs during boreal spring (Philander 1983; Hirst 1986). However, this suggestion cannot explain why the onset does not happen during the similarly unstable summer season, and does not provide an explicit explanation for the locking of ENSO's peak time to the end of the year. The coincidence of ENSO's peak time with the least unstable time of year was noted by $\mathrm{CZ}$ who attributed it to dissipative forces that could then overcome the weak instability and terminate the event. However, this mechanism does not incorporate the ocean waves whose role in ENSO's termination is now believed to be quite dominant.

Tziperman et al. (1997) examined the mechanisms by which the seasonal cycle in the equatorial Pacific affects ENSO events, using the CZ model. They found the most dominant seasonal effect on ENSO's dynamics to be due to the variations in the wind divergence field, as determined by the seasonal motion of the ITCZ, through its effect on the atmospheric heating. The next-order seasonal effects were found to be due to the seasonality of the background SST and ocean upwelling velocity, and the corresponding mechanisms were analyzed. An additional physical insight into the seasonal-ENSO interaction could be gained if studies such as Tziperman et al. (1997) could be complemented with an approach to the same problem of seasonal-ENSO interaction from the point of view of equatorial ocean waves. The development of this wave dynamics perspective is the purpose of the present work.

In the following sections we first introduce a seasonally varying coupled instability strength into a simple delayed oscillator model and present the proposed mechanism for ENSO's phase locking (section 2). We then proceed to analyze the $\mathrm{CZ}$ model in order to further examine the mechanism proposed using the simpler delayed oscillator model (section 3), and we conclude in section 4.

\section{A delayed-oscillator mechanism for ENSO's phase locking}

According to the delayed oscillator mechanism (Suarez and Schopf 1988; Graham and White 1988; Battisti 1988; Cane et al. 1990; Munnich et al. 1991), the ENSO cycle exists due to a combination of equatorial ocean wave dynamics and an amplification by a coupled ocean-atmosphere instability (Philander 1983; Hirst 1986). Consider a weakening of the easterlies in the central equatorial Pacific, which causes warm water to shift from higher latitudes toward the equator, creating a warm perturbation at the equator and cold perturbations off the equator. The resulting deepening thermocline perturbation at the equator propagates eastward as a warm Kelvin wave, reaching the eastern boundary after about 1 month. Upon reaching the eastern boundary, the thermocline deepening signal induces a warm sea surface temperature (SST) perturbation, which further weakens the low-level easterly winds near the equator (Bjerknes 1969). The weakened easterly winds amplify the deepening waves, creating a positive feedback (i.e., a coupled ocean-atmosphere instability) that leads to a rapid warming in the eastern equatorial Pacific Ocean. The cold SST perturbations off the equator in the central Pacific excite upwelling Rossby waves, which travel westward, and are reflected at the western boundary as cold equatorial Kelvin waves. Amplified again by the atmospheric feedback, these cold waves reach the eastern Pacific about six months after the original wind perturbation, and terminate the El Niño event.

We use a simple delayed oscillator model in which the seasonal variations in the coupled instability strength are represented by a seasonally varying ocean-atmosphere coupling coefficient (Zebiak and Cane 1987; Cane et al. 1990; Munnich et al. 1991). The model equation, very similar to that of Tziperman et al. (1994), is written for $h$, the nondimensionalized deviation of the thermocline depth at the eastern Pacific Ocean from its monthly mean, as a function of time, $t$ : 

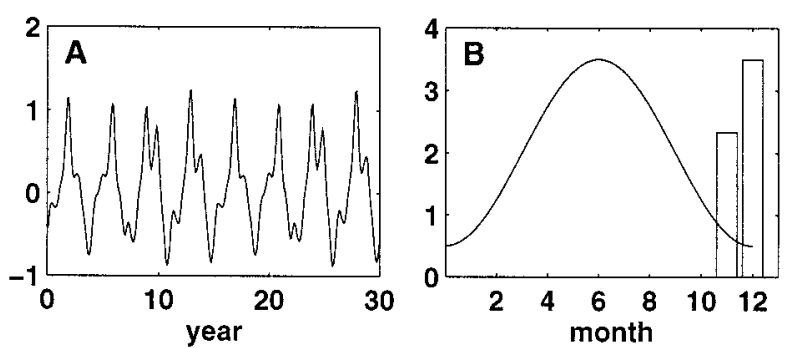

FIG. 2. (a) A short segment of the time series for $h(t)$, the solution to (1). (b) The coupling coefficient $\kappa(t)$ as a function of month (continuous line). Also shown is a scaled histogram of number of occurrences of peaks of events per month, during a 512-yr integration, showing that all events happen during Nov and Dec, near the minimum of $\kappa(t)$. The Dec bar corresponds to 81 events.

$$
\begin{aligned}
\frac{d h}{d t}= & b \mathcal{A}\left[\kappa\left(t-\tau_{1}\right) h\left(t-\tau_{1}\right)\right] \\
& -c \mathcal{A}\left[\kappa\left(t-\tau_{2}\right) h\left(t-\tau_{2}\right)\right]-d h(t) .
\end{aligned}
$$

The first term on the right-hand side is due to the Kelvin wave emanated from the central Pacific at time $t-\tau_{1}$ and reaching the eastern Pacific about one month later, at time $t$. The second term represents the first mode baroclinic Rossby wave starting in the central Pacific at time $t-\tau_{2}$ and reaching the eastern Pacific after about six months, at time $t$. The third represents various dissipation mechanisms. The monotonically increasing nonlinear function $\mathcal{A}$ models the nonlinear amplification of the ocean waves by the coupled ocean-atmosphere interaction, through the link between thermocline depth, SST, and the wind forcing (Munnich et al. 1991). The ocean-atmosphere coupling strength, corresponding to the strength of the coupled instability, is given by the seasonally varying $\kappa(t)$, which reaches its minimum at the end of the year. The model considers only wave reflection from the western boundary of the equatorial Pacific Ocean (Suarez and Schopf 1988; Graham and White 1988; Battisti 1988), and assumes that the waves are forced and amplified only at the basin center (Munnich et al. 1991).

The function $\mathcal{A}$ is a hyperbolic tangent modified by a straight-line segment inserted near the origin, as in Eqs. (9), (10), and (12) of Munnich et al. (1991):

$$
\begin{gathered}
\mathcal{A}[\kappa(t) h(t)] \\
\quad\left\{\begin{array}{l}
b_{+}+\frac{b_{+}}{a_{+}}\left\langle\tanh \left\{\frac{a_{+}}{b_{+}} \kappa(t)\left[h(t)-h_{+}\right]\right\}-1\right\rangle \\
\text { for } h>h_{+} \\
\quad \text { for } h_{-}<h<h_{+}, \\
b_{-}+\frac{b_{-}}{a_{-}}\left\langle\tanh \left\{\frac{a_{-}}{b_{-}} \kappa(t)\left[h(t)-h_{-}\right]\right\}-1\right\rangle \\
\text { for } h<h_{-},
\end{array}\right.
\end{gathered}
$$

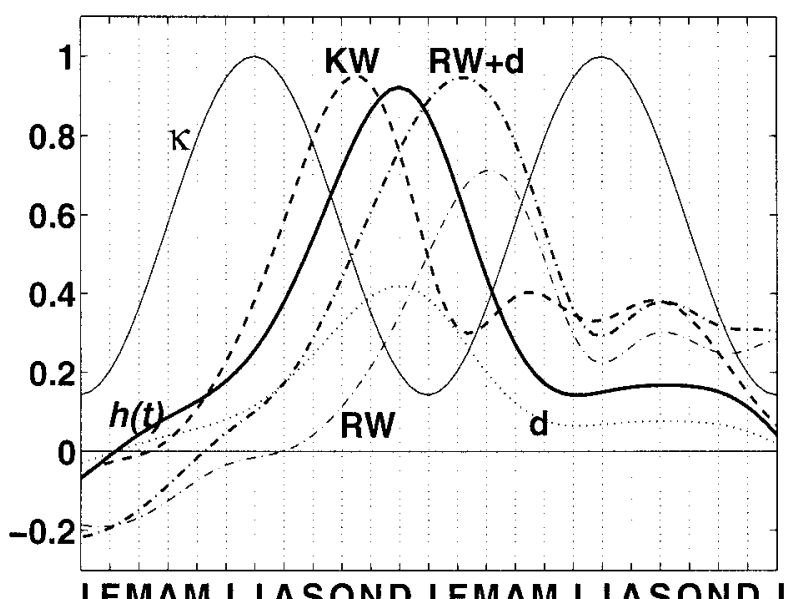

FIG. 3. An event from the solution of the delayed-oscillator equation (1) [curve scaled by 1.26 and marked $h(t)$ ], together with the seasonally varying coupling coefficient (curve scaled by a factor of 3.5 , and marked $\kappa$ ) and the amplitude of the various terms in the equation (scaled by 0.015 ). The curve marked KW is of the Kelvin wave term (first term on the rhs in 1); RW denotes the Rossby wave term (second term); d denotes the dissipation (third term); the curved marked RW $+\mathrm{d}$ is the sum of the Rossby and dissipation terms. The $x$ axis is in months.

where $h_{+}=b_{+}\left(a_{+}-1\right) /\left(\kappa a_{+}\right) ; h_{-}=b_{-}\left(a_{-}-1\right) /\left(\kappa a_{-}\right)$. The coupling coefficient, $\kappa(t)=\kappa_{0}+\Delta \kappa \sin \left(\omega_{a} t\right)$, representing the coupled instability strength, determines the slope of $\mathcal{A}[\kappa(t) h(t)]$ near the origin, at $h=0$. This slope determines in this model the strength of the amplification of the ocean waves by the coupled instability. The parameters specifying our model (1) and (2), that were used to obtain the solution analyzed in Figs. 2 and 3 , are $b_{+}=3 ; b_{-}=-1 ; a_{+}=1 ; a_{-}=1 \kappa_{0}=2 ; \Delta \kappa$ $=1.5 ; \tau_{1}=1.15$ months; $\tau_{2}=5.75$ months; $b=1 /$ 120 day $^{-1} ; c=1 / 160$ day $^{-1} ; d=1 / 190$ day $^{-1} ; \omega_{a}=$ $2 \pi \mathrm{yr}^{-1}$. These parameters were chosen to obtain satisfactory events in this simple model. We do not attempt to justify the quantitative choice of the various parameters, as such a more quantitative verification of the proposed mechanism is done in the next section using the more complete $\mathrm{CZ}$ model.

Neelin and Jin (1993) suggested that delayed oscillator equations such as (1) may also represent their mixed SST-wave dynamics mode (argued to be a more relevant dynamical regime of the actual equatorial $\mathrm{Pa}-$ cific). This correspondence between the mixed mode dynamics, the delayed oscillator mechanism, and a "recharge oscillator" mechanism was recently also demonstrated by Jin (1997). Note that in the mixed SSTwave dynamics mode (Neelin and Jin 1993) interpretation of (1), the time delays correspond more generally to ocean adjustment times rather than to the waves' travel times. For the sake of definiteness, we adopt the wave dynamics (delayed oscillator) perspective in the following, but the interpretation of our proposed mechanism in terms of the mixed mode dynamics or the 
recharge oscillator mechanism of Jin (1997) should not be difficult.

A time series of $h(t)$ from the solution of the model (1) is analyzed in Fig. 2, demonstrating that model events tend to peak when the coupling coefficient $\kappa(t)$ is at its minimum. We found this feature of the solution to be extremely robust with respect to all of the delayed model parameters. We now proceed to provide a physical explanation for the robust phase locking of ENSO events in this delayed oscillator model.

At the peak of an ENSO event in this model, the time derivative of $h(t)$ vanishes, $d h / d t=0$. This occurs in (1) when the warming trend due to the Kelvin wave term balances the cooling trend due to the Rossby term plus the dissipation. Figure 3 displays the various terms in (1) for an event that peaks in December, when the coupling coefficient $\kappa(t)$ is close to its minimum value. The delay $\tau_{1}$ of the Kelvin wave term is quite short, about one month. At the peak time $\left(t_{\text {peak }}\right)$, therefore, the Kelvin wave term in Eq. (1) is a function of a large thermocline depth anomaly $h\left(t_{\text {peak }}-\tau_{1}\right)$ one month before peak time, times the small value obtained by the coupling coefficient in November, $\kappa\left(t_{\text {peak }}-\tau_{1}\right)$. The delay in the Rossby wave term is longer, about six months. Therefore, the Rossby wave term evaluated at the peak time is a function of the small thermocline depth anomaly six months prior to the peak of the event, $h\left(t_{\text {peak }}-\tau_{2}\right)$, times the large value of coupling coefficient around spring time, $\kappa\left(t_{\text {peak }}-\tau_{2}\right)$. Thus the strongly amplified Rossby wave term, together with the dissipation term, may balance the weakly amplified Kelvin wave term, leading to $d h / d t=0$ and thus to the peaking of $h(t)$ as shown in Fig. 3.

The above balance of terms could not have occurred had the event peaked during the summer months, when the coupling coefficient is maximal. In that case, both the thermocline depth anomaly $h\left(t_{\text {peak }}-\tau_{1}\right)$ and the coupling coefficient $\kappa\left(t_{\text {peak }}-\tau_{1}\right)$ appearing in the Kelvin wave term would be near their respective maxima. On the other hand, both $h\left(t_{\text {peak }}-\tau_{2}\right)$ and $\kappa\left(t_{\text {peak }}-\tau_{2}\right)$ from the Rossby wave term would have been small. The peaktime balance $d h(t) / d t=0$ could not be satisfied in this case. While the above mechanism explains why events in the simple delayed oscillator model peak during the winter and not during summer, it does not explain why they peak in November and December and not in January, which is also characterized by a small $\kappa(t)$ (Fig. 2). This detail may be more sensitive to the model parameter choices and it seems that explaining the simple model's solution at such level of detail may not significantly add to our understanding of the locking of actual ENSO events.

Following the delayed oscillator theory, the ENSO cycle is viewed in the proposed mechanism as a continuous succession of Kelvin and Rossby waves whose amplitudes are related to that of the ENSO event at the time when the waves are excited. The waves are amplified by the seasonally varying coupled instability. The warm Kelvin wave arriving at the eastern boundary at the peak time has a large amplitude because it was emanated a month prior to the event peak time, during early winter, when the event amplitude was fairly large. The cold Rossby wave arriving at the eastern boundary at the peak time, after reflecting from the western boundary (as a cold Kelvin wave), was weaker when emanated 6 months prior to the peak time, during the summer, because the event amplitude was still weak. However, these weak Rossby waves are amplified along their path by the strong summertime coupled instability. (In our simplified delayed oscillator model, all the amplification is assumed to be instantaneous at the waves' creation time at the center of the basin). The peak time of the events is set by the dynamics to allow a balance between the warming and cooling trends due to warm Kelvin and cold Rossby waves. This balance is obtained because the warming trend due to the large amplitude Kelvin wave, amplified by a weak wintertime coupled ocean-atmosphere instability, balances the cooling trend due to weak Rossby waves, amplified by a strong summertime coupled instability.

The Rossby waves are expected to be damped by a partial reflection at the western boundary, be exposed to dissipation effects over a longer time than the Kelvin waves, are less efficiently excited by the wind forcing, and suffer larger dispersion effects. Thus the choice $b$ $>c$ in (1). The Rossby term can therefore balance the Kelvin term, leading to the event peak time in spite of these damping effects, only through the differential amplification of the Rossby and Kelvin waves by the seasonally varying coupled instability strength.

\section{Seasonal ocean-atmosphere coupling strength in the Cane-Zebiak model}

In an effort to test the proposed mechanism for ENSO's phase locking, we analyzed the coupled oceanatmosphere model of Zebiak and Cane (1987). Two different analyses are presented here, the purpose of both being to quantify the seasonal variations in the strength of the coupled ocean-atmosphere instability. This instability strength may be considered, according to the point of view presented in Zebiak and Cane (1987), as equivalent to a coupling strength between the ocean and the atmosphere. The seasonal variation in the coupling strength that is calculated in this section, in turn, relates directly to that postulated in the previous section, were it served as the base of the proposed mechanism for ENSO's phase locking within the idealized delayed oscillator picture. The first analysis presented here is based on a stability analysis of the $\mathrm{CZ}$ model using a tangent linear model and singular vector analysis (Xue et al. 1994). The second analysis is an explicit calculation of the seasonal amplification of the equatorial ocean modes in the $\mathrm{CZ}$ model. Both analyses demonstrate that the end of the year is the time of weakest coupled instability, 

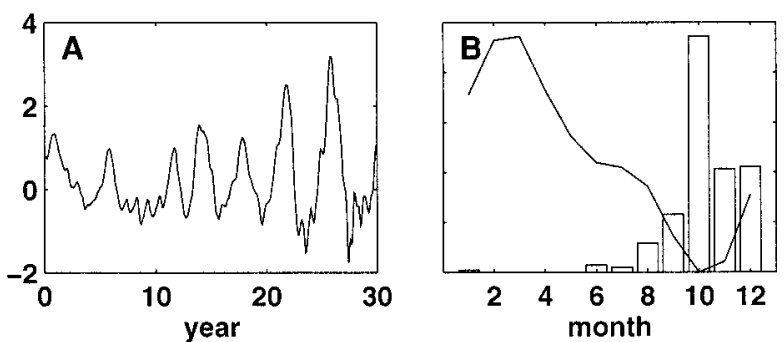

FIG. 4. (a) A segment of the time series for the CZ coupled oceanatmosphere ENSO model NINO3 index ( $y$ axis in ${ }^{\circ} \mathrm{C}$ ). (b) A scaled histogram of the number of $\mathrm{CZ}$ model ENSO events peaking per month of the calendar year during a 1024-yr-long model integration (the October bar corresponds to 98 events). A monthly instability index for the $\mathrm{CZ}$ model is shown by the continuous line.

as required by the mechanism for ENSO's phase locking presented in the previous section.

Figure 4 shows that most events in the $\mathrm{CZ}$ model peak during October-December. The same period is also when an index of the seasonal coupled instability strength in this model obtains its minimum (solid line in Fig. 4b), consistent with our proposed mechanism. The shown instability index is the increase in the NINO3 index during a 6-month model run, due to a perturbation to the initial conditions, as a function of the month in which the initial perturbation was inserted. The spatial structure of the perturbation to the initial conditions is of the first singular vector of the tangent linear model constructed for the $\mathrm{CZ}$ model in a reduced EOF space (Xue et al. 1994). The first singular vector, in turn, leads to a maximal energy growth in the tangent linear model during a 6-month integration. The use of the growth rate obtained over a 6-month period is a more accurate representation of the averaged instability rate seen by the ocean wave packets during their transit time than in the simpler model (1), where the ocean-atmosphere interaction is assumed to occur instantaneously in the central Pacific.

The second analysis using the $\mathrm{CZ}$ model we present here is based on an explicit calculation of the seasonal amplification of equatorial ocean modes, as follows. The model was first run for $400 \mathrm{yr}$, saving the time history of the upper-ocean layer thickness $h(x, y, t)$ and the zonal velocity $u(x, y, t)$. These are used to form the nondimensional variables $q(x, y, t)=h / H_{0}+u / c$ and $r(x, y$, $t)=h / H_{0}-u / c$, where $H_{0}$ is the equivalent depth and $c$ is the gravity wave speed. Next, these nondimensional variables are decomposed into equatorial ocean modes as explained in Gill (1983) and Battisti (1988, appendix B):

$$
\begin{aligned}
& q(x, y, t)=\sum_{m=0}^{\infty} q_{m}(x, t) \psi_{m}(y) \\
& r(x, y, t)=\sum_{m=0}^{\infty} r_{m}(x, t) \psi_{m}(y),
\end{aligned}
$$

where

$$
\begin{aligned}
q_{m}(x, t)= & \int d y q(x, y, t) \psi_{m}(y), \\
\psi_{m}(y)= & \left(2^{m} m ! \pi^{1 / 2}\right)^{-1 / 2} \exp \left(y^{2} / 2\right) \\
& \times \frac{d^{m}}{d y^{m}}\left[\exp \left(-y^{2}\right)\right](-1)^{m} .
\end{aligned}
$$

The $m$ th mode is denoted $q_{m}(x, t)$, where $q_{0}=K(x, t)$ is the equatorial Kelvin wave, $q_{1}$ is the Yanai wave, and $q_{2}=R_{1}(x, t)$ is the first Rossby mode. The wave speed of mode $q_{m+1}$ [Battisti's Eq. (B5)] is $c^{m+1}=c /(2 m+$ $1)$. The first Rossby mode $(m=2)$ is $q_{2}(x, t)$ and it therefore propagates at a speed $c / 3$, while the Kelvin wave propagates at a speed $c$.

Our objective is to calculate the amplification of the Kelvin and first Rossby modes along their trajectories. We examine the amplification of a given mode by following it along the wave characteristic $[x(t), t]=\left[x_{0}\right.$ $\left.+c^{m} \times\left(t-t_{0}\right), t\right]$, where $\left(x_{0}, t_{0}\right)$ are the initial time and location from which to follow the mode, and $c^{m}$ is its speed. For the Rossby modes, $x_{0}$ is near the center of the basin, while for the Kelvin mode, $x_{0}$ is at the western boundary.

The instantaneous amplification of the $m$ th mode along its trajectory at a given time is

$d A\left(t-t_{0}, m\right.$, month $)=\left\langle\frac{1}{q_{m}(x, t)} \frac{d q_{m}[x(t), t]}{d t}\right\rangle_{m o n t h}$,

where $x(t)=x_{0}+c^{m} \times\left(t-t_{0}\right)$ is the location along the mode trajectory, and the average \langle\rangle$_{\text {month }}$ is taken over all modes that start from $x_{0}$ at a given month. Next, given $d A$, we can calculate the total amplification along a trajectory as

$$
\begin{aligned}
& A\left(t-t_{0}, \text { m, month }\right) \\
& \quad=\exp \left[\int_{0}^{t} d A\left(t^{\prime}-t_{0}, \text { m, month }\right) d t^{\prime}\right] .
\end{aligned}
$$

To reiterate, $A\left(t-t_{0}, m=2\right.$, month $\left.=3\right)$ represents the averaged total amplification of the first Rossby modes $(m=2)$ that start traveling westward from the middle of the basin during March (month = 3). The averaged amplification represents the averaged ratio between the amplitude of this mode at a time $t-t_{0}$ after starting from the middle of the basin, and its initial amplitude at $t_{0}$. The average in this case is over all packets of the first Rossby mode starting from near the center of the basin during March. Similarly, $A\left(t-t_{0}\right.$, $m=0$, month $=3$ ) represents the averaged amplification of the Kelvin mode packets $(m=0)$ that started at the western boundary during the month of March, traveling toward the middle of the basin.

Figure 5a shows a contour plot of the Kelvin mode amplification $A\left(t-t_{0}, m=0\right.$, month $)$ as a function of both the starting month (horizontal axis) and the travel time from the western Pacific (vertical axis). The Kelvin mode was followed in this case over a distance of 0.6 


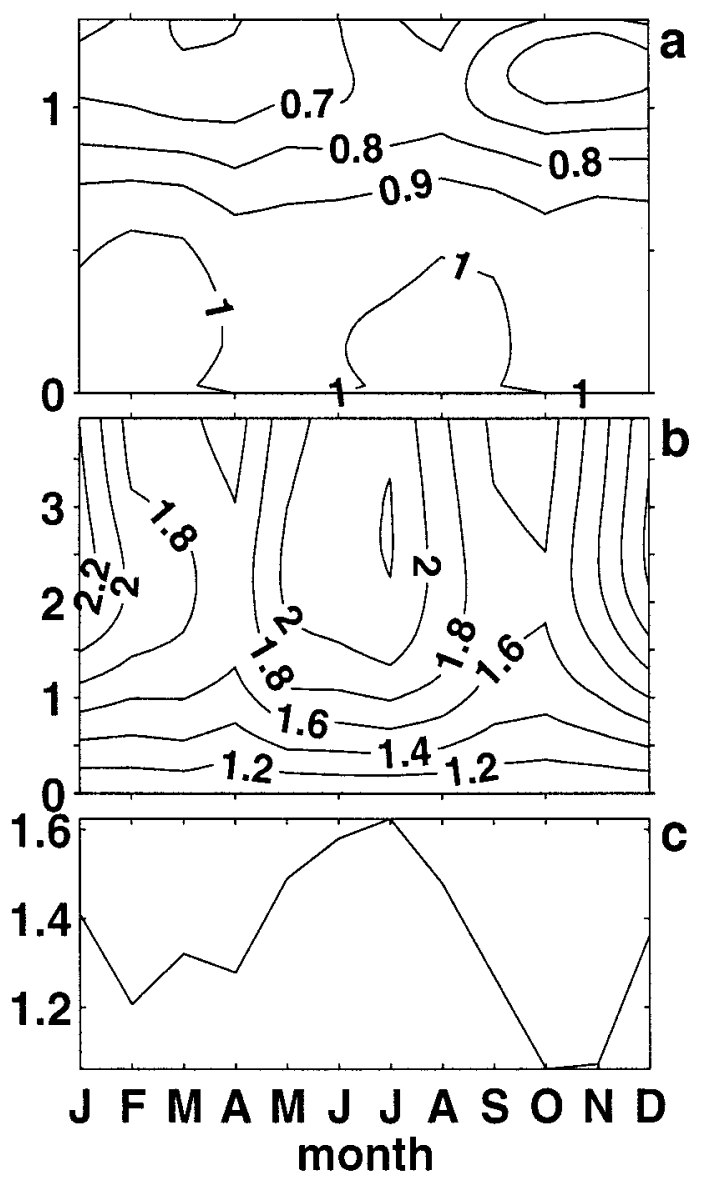

FIG. 5. (a) The averaged amplification of Kelvin modes from their starting point at the western boundary as they travel eastward, as function of the starting month ( $x$ axis) and the travel time ( $y$ axis). (b) The averaged amplification of first Rossby modes from their starting point in the central Pacific as they travel westward, as function of the starting month ( $x$ axis) and the travel time ( $y$ axis). (c) The total amplification of a mode that starts propagating as a Rossby mode from the central Pacific at the month given on the $x$ axis, gets reflected at the western boundary as a Kelvin wave, and reaches the middle of the basin again as a Kelvin mode.

times the basin width, from the western boundary toward the center of the basin. Figure $5 b$ shows a similar contour plot of the first Rossby mode amplification, $A(t$ $-t_{0}, m=2$, month), as a function of both the starting month and the travel time from the central Pacific. Again the Rossby modes were followed over a distance of 0.6 times the basin width toward the western boundary. Figure 5c combines the separate Kelvin and Rossby amplifications into a total averaged amplification of a Rossby mode that starts near the center of the basin at a given month, gets reflected at the western boundary as a Kelvin wave, and reaches the original starting point of the Rossby mode near the center the basin again. This total amplification is calculated as

$$
\begin{aligned}
A_{T}(\text { month })= & A\left(\Delta t_{\text {Rossby }}, m=2, \text { month }\right) \\
& \times A\left(\Delta t_{\text {Kelvin }}, m=0, \text { month }_{\text {reflect }}\right),
\end{aligned}
$$
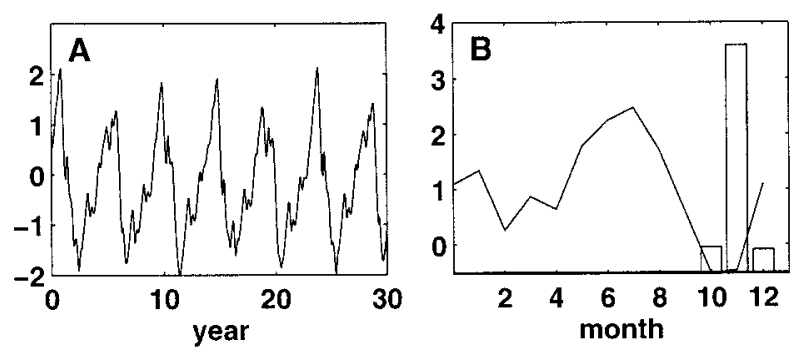

FIG. 6. As in Fig. 2 except that the shape of the coupling strength is taken from the explicit calculation of seasonal mode amplification in the $\mathrm{CZ}$ model, as seen in Fig. 5c.

where $\Delta t_{\text {Rossby }}$ and $\Delta t_{\text {Kelvin }}$ are the travel times of the Rossby mode from the starting point near the center of the basin to the west boundary, and of the Kelvin mode from the western boundary to the Rossby mode's starting point; month $_{\text {reflect }}$ is the month at which the Rossby mode, starting at month $=$ month , is reflected into a Kelvin mode. The amplifications in Fig. 5 are based on a 400 -yr average.

Figure 5c shows that modes that start at the center of the basin as Rossby modes during June and July are amplified most significantly (by a factor of about 1.6) by the time they return to their starting point near the center of the basin as Kelvin modes. Modes that start during October and November are not amplified, and return to about their initial amplitude. The total amplification seen in Fig. 5c is the combined effect of the separate amplification (and damping) of Rossby and Kelvin modes, as seen in the contour plots of Figs. 5b and $5 \mathrm{a}$. This calculation of the total amplification ignores the fact that the reflection at the western boundary is not completely efficient, but still demonstrates that the end of the year is the least unstable time for the traveling equatorial ocean modes, while the early summer is the most unstable time.

The total seasonal amplification of the ocean modes, $A_{T}$ (month), as shown in Fig. $5 \mathrm{c}$ is, in fact, precisely what $\kappa(t)$ in the Rossby wave term in Eq. (1) stands for in our simple delayed oscillator model analyzed in the previous section. We can therefore use the shape of the total amplification shown in Fig. 5c as a coupling strength in the simple delayed oscillator model of the previous section. When this is done (Fig. 6), the events in the simple delayed oscillator model peak around October-December, as in the fuller CZ model. The results of the delayed oscillator model shown in Fig. 6 are obtain by using for the coupling strength $\kappa(t)$ in the Rossby wave term of (1) the exact shape found from the CZ model and seen in Fig. 5c. The amplitude and mean of the monthly coupling strength in the delayed oscillator model, $\boldsymbol{\kappa}(\mathrm{m})$, were tuned to allow for selfsustained oscillations in the simple delayed oscillator model, by setting 


$$
\begin{gathered}
\kappa(m)=C_{1} \frac{\kappa_{\mathrm{cz}}(m)-\min \left(\kappa_{\mathrm{cz}}\right)}{\max \left(\kappa_{\mathrm{cz}}\right)-\min \left(\kappa_{\mathrm{cz}}\right)}+C_{2}, \\
m=1 \ldots 12,
\end{gathered}
$$

where $\kappa_{\mathrm{cz}}(m)$ is the average monthly amplification in the $\mathrm{CZ}$ model, seen in Fig. $5 \mathrm{c}$, and the constants $C_{1}=$ 3 and $C_{2}=1$ determine the amplitude and mean of the Rossby amplification term used in the model (1). The coupling strength in the Kelvin wave term of (1), representing the amplification (or damping) occurring over the less than 1 month travel time from the center of the basin to the eastern boundary, is taken as uniform in time and equal to 0.9 . The locking of the event peaks to the end of the year, as seen in Fig. 6, is extremely robust to the specified amplitude and mean of the monthly coupling coefficient (i.e., to $C_{1}$ and $C_{2}$ ), as long as the amplitude $\left(C_{1}\right)$ of the seasonal variations of $\kappa(t)$ is sufficiently large for these seasonal variations to be of a dynamical significance.

The similarity of the phase locking of the ENSO event peaks in the fuller CZ model (Fig. 4) and in the simple delayed oscillator model using the explicit mode amplification calculated from the CZ model (Fig. 6), clearly strengthens the case for our proposed phase-locking mechanism. Note that there is a 1-month phase-lag between the peak time in the simple delayed oscillator when using the idealized $\kappa(t)$ (Fig. 2b) and when using the $\kappa(t)$ deduced from the CZ model (Fig. 6b). Similarly, there is 1-month lag between peak time in the full $\mathrm{CZ}$ model and in the delayed oscillator model using the $\kappa(t)$ deduced from the CZ model. Clearly our proposed mechanism for the timing of the warm event peak time, being simple as well as somewhat qualitative, cannot account for such small differences in the peak time.

The seasonal amplification of the first mode Rossby wave (Fig. 5b) shows a nonnegligible semiannual signal (amplification in both summer and winter). Consequently, the total amplification of the combined Rossby and Kelvin modes (Fig. 5c) also shows a semiannual signal with local minima both at February-March and at October-November. The model events peak during October-November, when the amplification is at its all-year minimum. This minimum also follows a strong amplification maximum during June-July, which is significantly larger than the amplification maximum in December-January. Thus, the semiannual amplification signal is not dominant, and there is still a strong annual component to the total amplification, resulting in the observed distribution of peak times of the model events.

While this explicit calculation of the seasonal mode amplification is quite encouraging, we would like to note some of its deficiencies. First, there is a nonnegligible sensitivity of the results in Fig. 5 to the total distance over which the modes are followed. That is, if the Rossby and Kelvin modes are followed over a distance of 0.5 or 0.7 times the basin distance rather than 0.6 , the most amplified and most damped times of year may shift by a month or two. This sensitivity is certainly a reason for some concern, and future work will need to further examine the robustness of the proposed mechanism both using observations and also using further analysis of the CZ model. Still, the general shape seen in Fig. 5c is reasonably robust. A second cautionary note concerns the rms variability around the mean amplifications shown in Figs. 5a-c. The mean shown in these figures is most robust (based on a 400-yr average, where a 200yr average would already suffice for a stable estimate of the mean). Still, the rms variability around this mean is of about the same magnitude as the mean itself. This indicates that in some years the traveling modes see a significantly different seasonal amplification than that of Figs. 5a-c. This, according to our mechanism, might have resulted in less robust locking of the events in the $\mathrm{CZ}$ model to the end of the year than actually seen in Fig. 4. This is an indication that the actual ENSO phaselocking mechanism in the $\mathrm{CZ}$ model, and certainly in observed ENSO events, is likely to be more complex than that of the highly idealized delayed oscillator model of the previous section.

\section{Conclusions}

A simple delayed oscillator mechanism was proposed for the locking of ENSO peak time, as measured by the maximum basinwide east Pacific warming, to the end of the calendar year. It was shown that within the idealized delayed-oscillator picture, the peak time of the events is set by the dynamics to allow a balance between the warming and cooling trends due to warm Kelvin and cold Rossby waves. This balance is obtained because the warming trend due to the large amplitude Kelvin waves, amplified by a weak wintertime coupled ocean-atmosphere instability, balances the cooling trend due to weak Rossby waves, amplified by a strong summertime coupled instability.

While most observed ENSO events tend to peak toward the end of the year, there are some notable exceptions (e.g., the events of 1957 or 1977). In particular, the sustained warming during the beginning of the 1990s has been quite unusual, and did not always peak toward the end of the year. The ideas presented here may offer a conceptual framework for examining these deviations from the normal pattern of ENSO's evolution. For example, our mechanism assumes that the event amplitude is quite weak a few months prior to the peak. A sufficiently strong event can affect the background stability to a degree that it can sustain itself independently of the mean seasonal conditions (Philander 1983). Such a long-lasting event can avoid our dynamical constraint and may not peak toward the end of the year. In addition, the constraint on the timing of ENSO events may be broken by changes to the mean seasonal conditions in the equatorial Pacific. There is indeed evidence $(\mathrm{Gu}$ et al. 1997) that such changes have occurred over the past 
few years, possibly resulting in the unusual recent ENSO events.

The proposed mechanism was demonstrated here using a highly simplified delayed oscillator model, and further examined using different analyses of the Zebiak and Cane (1987) model. It is well understood now that the delayed oscillator paradigm is an oversimplification of the actual ENSO dynamics (e.g., Picaut et al. 1997). Future work on the wave dynamics perspective presented here for the seasonal-ENSO interaction mechanism will need to apply similar ideas to the different mechanisms that have been proposed for the ENSO cycle [e.g., the mixed SST-wave dynamics mode of Neelin and Jin (1993) and the recharge oscillator of Jin (1997)].

Note that in Fig. 1 we have only plotted events up to the 1982 event. The character of the more recent ENSO events has been somewhat different, with western Pacific or basin-scale warming occurring ahead of the major eastern coastal warming. This may also have had some implication regarding the character of the event's phase locking to the seasonal cycle. In addition, one might expect the proposed mechanism to result in locking of cold La Niña events to the seasonal cycle, but such phase locking does not show up as a prominent feature for the cold events of ENSO. The detailed investigation of such issues requires the use of additional data and is perhaps better left for future studies.

There are clearly still quite a few open questions regarding the applicability of the ideas presented here to the actual equatorial Pacific. Model events in the simple delayed-oscillator model and even in the CZ model are, of course, different in some important regards from their observed counterparts. One particularly important difference between the $\mathrm{CZ}$ model parameterizations and the actual equatorial Pacific has to do with the parameterization of atmospheric heating, as pointed out by an anonymous reviewer of this paper:

Typically, the main equatorial wind anomalies in ENSO are driven by deep convection in the equatorial central western Pacific between about $160^{\circ} \mathrm{E}$ and $160^{\circ} \mathrm{W}$ (Fig. 5 of Deser and Wallace 1990). The deep convection does not occur farther to the east, despite the larger SST anomalies there, because the total water temperature is too low there-Gadgil et al. (1984) and Graham and Barnett (1987) showed that the SST must be at least $28^{\circ} \mathrm{C}$ before convection occurs. But the model heating has no such necessary condition and model heating will occur all over the central-eastern Pacific. The model heating will thus be strongly influenced by the presence of the ITCZ (Tziperman et al. 1997), since this occurs most strongly in the central-eastern equatorial pacific, but the real-world ENSO heating will not.

These important differences between the mechanism of seasonal amplification in the models used here and in reality do not invalidate the essence of the mechanism proposed here. As long as this seasonal amplification is weakest during winter and strongest during spring-sum- mer, the mechanism proposed here may be relevant to the locking of observed ENSO events to the calendar year. We conclude that the mechanism proposed here for the locking of ENSO events to the seasonal cycle is quite speculative at this stage, and requires further validation using both more realistic models and additional data analysis, perhaps via data assimilation into fuller coupled models.

Acknowledgments. We thank Steve Griffies for useful discussions and Larry Rosen for his help. Very detailed and constructive comments from Ping Chang and from anonymous reviewers are greatly appreciated. This work is partially supported by a grant from NOAA through the Consortium for Climate Research, and by a grant from the Israeli-U.S. binational science foundation.

\section{REFERENCES}

Battisti, D. S., 1988: Dynamics and thermodynamics of a warming event in a coupled tropical atmosphere-ocean model. J. Atmos. Sci., 45, 2889-2919.

_, and E. S. Sarachik, 1995: Understanding and predicting ENSO. Rev. Geophys., 33 (Suppl.), 1367-1376.

Bjerknes, J., 1969: Atmospheric teleconnections from the equatorial Pacific. Mon. Wea. Rev., 97, 163-172.

Blumenthal, M. B., 1991: Predictability of a coupled ocean-atmosphere model. J. Climate, 4, 766-784.

Bottomley, M., C. K. Folland, J. Hsiung, R. E. Newell, and D. E. Parker, 1990: Global Ocean Surface Temperature Atlas "GOSTA." Meteorological Office and the Massachusetts Institute of Technology, 20 pp. and 313 plates.

Cane, M. A., M. Munnich, and S. E. Zebiak, 1990: A study of selfexcited oscillations of the tropical ocean-atmosphere system. Part I: Linear analysis. J. Atmos. Sci., 47, 1562-1577.

Chang, P., B. Wang, T. Li, and L. Ji., 1994: Interactions between the seasonal cycle and the Southern Oscillation-Frequency entrainment and chaos in a coupled ocean-atmosphere model. Geophys. Res. Lett., 21, 2817-2820.

—, L. Ji, B. Wang, and T. Li, 1995: Interactions between the seasonal cycle and El Niño-Southern Oscillation in an intermediate coupled ocean-atmosphere model. J. Atmos. Sci., 52, 23532372.

Chen, D., S. E. Zebiak, A. J. Busalacchi, and M. A. Cane, 1995: An improved procedure for El Niño forecasting: Implications for predictability. Science, 269, 1699-1702.

Deser, C., and J. M. Wallace, 1990: Large-scale atmospheric circulation features of warm and cold episodes in the tropical Pacific. J. Climate, 3, 1254-1281.

Gadgil, S., P. V. Joseph, and N. V. Joshi, 1984: Ocean-atmosphere coupling over monsoon regions. Nature, 312, 141-143.

Gill, A. E., 1983: An estimation of sea-level and sea-current anomalies during the $1972 \mathrm{El}$ Niño and consequent thermal effects. J. Phys. Oceanogr., 13, 586-605.

Goswami, B. N., and J. Shukla, 1991: Predictability of a coupled ocean-atmosphere model. J. Climate, 4, 107-115.

Graham, N. E., and T. P. Barnett, 1987: Sea surface temperature, surface wind divergence, and convection over tropical oceans. Science, 238, 657-659.

_ of the Pacific ocean-atmosphere system. Science, 240, 12931302 .

Gu, D., S. G. H. Philander, and M. J. McPhaden, 1997: The seasonal cycle and its modulation in the eastern tropical Pacific Ocean. J. Phys. Oceanogr., 27, 2209-2218. 
Hirst, A. C., 1986: Unstable and damped equatorial modes in simple coupled ocean-atmosphere models. J. Atmos. Sci., 43, 606-630.

Jin, F.-F., 1997: An equatorial ocean recharge paradigm for ENSO Part I: Conceptual model. J. Atmos. Sci., 54, 811-829.

- D. Neelin, and M. Ghil, 1994: ENSO on the devil's staircase. Science, 264, 70-72.

Latif, M., T. P. Barnett, M. A. Cane, M. Flugel, N. E. Graham, H von Storch, J.-S. Xu, and S. E Zebiak, 1994: A review of ENSO prediction studies. Climate Dyn., 9, 167-179.

Munnich, M., M. A. Cane, and S. E. Zebiak, 1991: A study of selfexcited oscillations of the tropical ocean-atmosphere system. $J$. Atmos. Sci., 48, 1238-1248.

Neelin, J. D., and E.-F. Jin, 1993: Models of interannual tropical ocean-atmosphere interaction-A unified view. Part II: Analytical results in the weak-coupling limit. J. Atmos. Sci., 50, 35043522.

Philander, S. G., 1983: El Niño Southern Oscillation phenomena. Nature, 302, 295-301.

Picaut, J., F. Masia, and Y. du Penhoat, 1997: An advective-reflective conceptual model for the oscillatory nature of the ENSO. Science, 277, 663-666
Rasmusson, E., and T. Carpenter, 1982: Variations in tropical sea surface temperature and surface wind fields associated with the Southern Oscillation/El Niño. Mon. Wea. Rev., 110, 354-384.

Suarez, M. J., and P. S. Schopf, 1988: A delayed action oscillator for ENSO. J. Atmos. Sci., 45, 3283-3287.

Tziperman, E., L. Stone, M. Cane, and H. Jarosh, 1994: El Niño chaos: Overlapping of resonances between the seasonal cycle and the Pacific Ocean-atmosphere oscillator. Science, 264, 7274.

— the seasonal cycle in an ENSO prediction model as explained by the quasi-periodicity route to chaos. J. Atmos. Sci., 52, 293306.

- S. Zebiak, and M. A. Cane, 1997: Mechanisms of seasonalENSO interaction. J. Atmos. Sci., 54, 61-71.

Vallis, G. K., 1988: Conceptual models of El Niño and the Southern Oscillation. J. Geophys. Res., 93C, 13 979-13 991.

Xue, Y., M. A. Cane, S. E. Zebiak, and M. B. Blumenthal, 1994: On the prediction of ENSO: A study with a low-order Markov model. Tellus, 46A, 512-528.

Zebiak, S. E., and M. A. Cane, 1987: A model El Niño-Southern Oscillation. Mon. Wea. Rev., 115, 2262-2278. 